\title{
Enalapril and the VEGFA gene: Personalized medicine in hypertension therapy
}

\author{
Firoj Kumar Sahoo ${ }^{1}$
}

Received: 24 October 2015 / Accepted: 16 November 2015 /Published online: 23 November 2015

(C) Springer-Verlag Berlin Heidelberg 2015

Dear editor,

Polymorphisms in the vascular endothelial growth factor A (VEGFA) gene and their effect on antihypertensive responses to enalapril were studied by Oliveira-Paula et al., and the results were published recently [1]. With the introduction of modern technologies to determine genetic variations in an individual, the concept of personalized medicine receives increasing acceptance among health care personnel [2].

The authors' effort to identify the pharmacogenomic association between the VEGF gene and the antihypertensive response produced with enalapril is highly appreciated as it might serve as the first step towards unraveling of the genetic puzzle of antihypertensive treatment. However, our major concern rests in the fact that hypertension is a multigenetic disease, with antihypertensive response governed by an intricate regulation of genes [3], and this association may be an oversimplification. Multiple genetic interactions can significantly confound the results. Addition of biological parameters like NO levels and bradykinin levels to correlate with the gene expression would have helped in formulating an association and rule out any significant interactions.

Moreover, the authors have not mentioned any details regarding the calculated sample size. In the discussion, the authors have mentioned in the shortcomings of the study that the sample size is low. Therefore, it can be assumed that sample size was calculated. Sample size calculation prior

Firoj Kumar Sahoo

firojpharmacology@gmail.com

1 Department of Pharmacology, JIPMER, Pondicherry, India to the study provides an estimate of the approximate number of subjects that need to be studied to provide significant results [4]. Without the sample size, it is difficult to judge the magnitude by which the study fell short. The study showed significant results with one polymorphism ( $\mathrm{g}-2578 \mathrm{C}>\mathrm{A}$ ) only in mean $\mathrm{BP}$ and in all parameters in the AGG haplotype. This result was not demonstrated in the $10 \mathrm{mg}$ enalapril group. Though g-1154 G $>$ A and g-634 G $>$ C did not show a significant difference in parameters, they did demonstrate a trend. Thus, it is interesting to retrospect that with an adequate sample size, they might have demonstrated significance too. Moreover, the subgrouping of the data might have further reduced the power. A retrospective power calculation would have provided a clear picture. Hence, power calculation should have been attempted.

Despite these slips, the article in our opinion did contribute to understanding the role of polymorphisms in the VEGFA gene in the antihypertensive responses to enalapril.

\section{References}

1. Oliveira-Paula GH, Lacchini R, Fontana V, Silva PS, Biagi C, TanusSantos JE (2015) Polymorphisms in VEGFA gene affect the antihypertensive responses to enalapril. Eur J Clin Pharmacol 71:949-957

2. Kashyap MV, Nolan M, Sprouse M, Chakraborty R, Cross D, Roby $\mathrm{R}$ et al (2015) Role of genomics in eliminating health disparities. J Carcinog 11:6. doi:10.4103/1477-3163.165158

3. Kunes J, Zicha J (2009) The interaction of genetic and environmental factors in the etiology of hypertension. Physiol Res 58:S33-S41

4. Noordzij M, Tripepi G, Dekker FW, Zoccali C, Tanck MW, Jager KJ (2010) Sample size calculations: basic principles and common pitfalls. Nephrol Dial Transplant Off Publ Eur Dial Transpl Assoc - Eur Ren Assoc 25:1388-1393 